\title{
Periodic Solutions and Slow Manifolds
}

\author{
Ferdinand Verhulst \\ Mathematisch Instituut \\ University of Utrecht \\ PO Box 80.010, 3508 TA Utrecht \\ The Netherlands
}

\begin{abstract}
After reviewing a number of results from geometric singular perturbation theory, we give an example of a theorem for periodic solutions in a slow manifold. This is illustrated by examples involving the van der Pol-equation and a modified logistic equation. Regarding nonhyperbolic transitions we discuss a 4-dimensional relaxation oscillation and also canardlike solutions emerging from the modified logistic equation with sign-alternating growth rates.
\end{abstract}

\section{Singular perturbations and slow manifolds}

In singular perturbations, certain attraction (or hyperbolicity) properties of the regular (outer) expansion play an essential part in the construction of the formal approximation. In the case of initial value problems, such a regular expansion is associated with the existence of a so-called slow manifold. In the actual constructions, the Tikhonov theorem provides a basic boundary layer property of the solution, leading naturally to a number of qualitative and quantitative results.

\subsection{The Tikhonov theorem}

The following result was obtained in 1952 by Tikhonov [18]:

Theorem 1.1

Consider the initial value problem

$$
\begin{aligned}
\dot{x} & =f(x, y, t)+\varepsilon \cdots, \quad x(0)=x_{0}, \quad x \in D \subset \mathbb{R}^{n}, t \geq 0, \\
\varepsilon \dot{y} & =g(x, y, t)+\varepsilon \cdots, \quad y(0)=y_{0}, \quad y \in G \subset \mathbb{R}^{m} .
\end{aligned}
$$

For $f$ and $g$, we take sufficiently smooth vector functions in $x, y$, and $t$; the dots represent (smooth and bounded) higher-order terms in $\varepsilon$.

a. We assume that a unique solution of the initial value problem exists and suppose this holds also for the reduced problem

$$
\begin{aligned}
& \dot{x}=f(x, y, t), \quad x(0)=x_{0}, \\
& 0=g(x, y, t),
\end{aligned}
$$


with solutions $\bar{x}(t), \bar{y}(t)$.

b. Suppose that $0=g(x, y, t)$ is solved by $\bar{y}=\phi(x, t)$, where $\phi(x, t)$ is a continuous function and an isolated root. Also suppose that $\bar{y}=\phi(x, t)$ is an asymptotically stable solution of the equation

$$
\frac{d y}{d \tau}=g(x, y, t)
$$

that is uniform in the parameters $x \in D$ and $t \in \mathbb{R}^{+}$.

c. $y(0)$ is contained in an interior subset of the domain of attraction of $\bar{y}=\phi(x, t)$ in the case of the parameter values $x=x(0), t=0$.

Then we have

$$
\begin{aligned}
& \lim _{\varepsilon \rightarrow 0} x_{\varepsilon}(t)=\bar{x}(t), \quad 0 \leq t \leq L, \\
& \lim _{\varepsilon \rightarrow 0} y_{\varepsilon}(t)=\bar{y}(t), \quad 0<d \leq t \leq L
\end{aligned}
$$

with $d$ and $L$ constants independent of $\varepsilon$.

The system

$$
\dot{x}=f(x, y, t), 0=g(x, y, t),
$$

is usually called the unperturbed, reduced or degenerate system.

In assumption (b), $t$ and $x$ are parameters and not variables. The idea is that during the fast motion of the variable $y$, the small variations of these parameters are negligible as long as the stability holds for values of the parameters $x \in D$ and $t \in \mathbb{R}^{+}$. For a discussion of the Tikhonov theorem see [22].

\subsection{Early results on periodic solutions}

An early example of a periodic solution theorem can be found in [9]. If the reduced system (1) has a hyperbolic $T$-periodic solution, then under certain additional conditions, the full system has a unique $T$-periodic solution.

Slightly more general results have been published in [1]. Hyperbolicity of the periodic solution of the reduced system plays an essential part in both papers and this prohibits application to the important case when the reduced system is Hamiltonian. Later extensions by students of Anosov deal to some extent with this problem.

A major technical problem was the absence of a theorem on the existence of a manifold of solutions (the so-called slow manifold), corresponding with the solutions of the reduced system (1). This complicated the existence problem of the theorems of that time enormously.

\subsection{The O'Malley-Vasil'eva expansion}

How do we use Tikhonov's theorem to obtain approximations of solutions of nonlinear initial value problems? The theorem does not state anything about the size of the boundary layer (the parameter $d$ in the theorem) or the timescales involved to describe the initial behaviour and the relative slow behaviour later on.

Asymptotic expansions are described as follows (for references see [22]): 


\section{Theorem 1.2}

(O'Malley-Vasil'eva)

Consider the initial value problem in $\mathbb{R}^{n} \times \mathbb{R}^{m} \times \mathbb{R}^{+}$

$$
\begin{aligned}
\dot{x} & =f(x, y, t, \varepsilon), x(0)=x_{0}, \quad x \in D \subset \mathbb{R}^{n}, t \geq 0, \\
\varepsilon \dot{y} & =g(x, y, t, \varepsilon), y(0)=y_{0}, \quad y \in G \subset \mathbb{R}^{m},
\end{aligned}
$$

where $f$ and $g$ can be expanded in powers of $\varepsilon$ to order $(m+1)$. Suppose that the requirements of Tikhonov's theorem have been satisfied and moreover that for the solution of the reduced system $0=g(x, \bar{y}, t, 0), \bar{y}=\phi(x, t)$ we have, with $\mu$ a constant independent of $\varepsilon$,

$$
\operatorname{Re} \mathrm{Sp} g_{y}(x, \bar{y}, t) \leq-\mu<0, x \in D, 0 \leq t \leq L .
$$

Then, for $t \in[0, L], x \in D, y \in G$, the formal approximation described above leads to asymptotic expansions of the form

$$
\begin{aligned}
& x_{\varepsilon}(t)=\sum_{n=0}^{m} \varepsilon^{n} a_{n}(t)+\sum_{n=1}^{m} \varepsilon^{n} \alpha_{n}\left(\frac{t}{\varepsilon}\right)+O\left(\varepsilon^{m+1}\right), \\
& y_{\varepsilon}(t)=\sum_{n=0}^{m} \varepsilon^{n} b_{n}(t)+\sum_{n=0}^{m} \varepsilon^{n} \beta_{n}\left(\frac{t}{\varepsilon}\right)+O\left(\varepsilon^{m+1}\right) .
\end{aligned}
$$

The constant $L$ that bounds the domain of validity in time is in general an $O(1)$ quantity determined by the vector fields $f$ and $g$. There are cases where $L$ extends to $\infty$.

An intermediate step in the analysis by O'Malley and Vasil'eva is an expansion of the form

$$
y=\phi(x, t)+\varepsilon y_{1}(x, t)+\varepsilon^{2} y_{2}(x, t)+\varepsilon^{3} \cdots .
$$

The expansion is derived from the fast equation and it is asymptotically valid on a timescale $O(1)$ outside the boundary layer in time where fast motion takes place.

\subsection{The slow manifold: Fenichel's results}

Tikhonov's theorem is concerned with the attraction, at least for some time, to the regular expansion that corresponds with a stable critical point of the boundary layer equation. The theory is quite general and deals with nonautonomous equations.

In the case of autonomous equations, it is possible to associate with the regular expansions $\sum_{n=0}^{m} \varepsilon^{n} a_{n}(t)$ and $\sum_{n=0}^{m} \varepsilon^{n} b_{n}(t)$, a manifold in phase-space and to consider the attraction properties of the flow near this manifold. Such questions were addressed and answered in a number of papers by Fenichel $(1971,1974,1977,1979)$, and other authors; the reader is referred to the survey papers [11] and [12]. See for an introduction also [22].

Consider the autonomous system

$$
\begin{aligned}
\dot{x} & =f(x, y)+\varepsilon \cdots, \quad x \in D \subset \mathbb{R}^{n}, \\
\varepsilon \dot{y} & =g(x, y)+\varepsilon \cdots, \quad y \in G \subset \mathbb{R}^{m} .
\end{aligned}
$$

In this context, one often transforms $t \rightarrow \tau=t / \varepsilon$ so that

$$
\begin{aligned}
& x^{\prime}=\varepsilon f(x, y)+\varepsilon^{2} \cdots, \quad x \in D \subset \mathbb{R}^{n}, \\
& y^{\prime}=g(x, y)+\varepsilon \cdots, \quad y \in G \subset \mathbb{R}^{m},
\end{aligned}
$$


where the prime denotes differentiation with respect to $\tau$.

As before, $y$ is called the fast variable and $x$ the slow variable. The zero set of $g(x, y)$ is given again by $y=\phi(x)$, which in this autonomous case represents a first-order approximation $M_{0}$ of the $n$-dimensional (slow) manifold $M_{\varepsilon}$. The flow on $M_{\varepsilon}$ is to a first approximation described by $\dot{x}=f(x, \phi(x))$.

Note that the assumption for the system to be autonomous is not essential for Fenichel's theory; it only facilitates the geometric interpretation.

In Tikhonov's theorem, we assumed asymptotic stability of the approximate slow manifold; in the asymptotic constructions we assume that the eigenvalues of the linearised flow near $M_{0}$, derived from the equation for $y$, have negative real parts only.

In geometric singular perturbation theory, for which Fenichel's results are basic, we only assume that all real parts of the eigenvalues are nonzero. In this case of a slow-fast system, the slow manifold $M_{\varepsilon}$ is called normally hyperbolic. A manifold is called hyperbolic if the local linearisation is structurally stable (real parts of eigenvalues all nonzero), and it is normally hyperbolic if in addition the expansion or contraction near the manifold in the transversal direction is larger than in the tangential direction (the slow drift along the slow manifold).

If $M_{0}$ is a compact manifold that is normally hyperbolic, it persists for $\varepsilon>0$ (i.e., there exists for sufficiently small, positive $\varepsilon$ a smooth manifold $M_{\varepsilon}$ close to $M_{0}$ ). Corresponding with the signs of the real parts of the eigenvalues, there exist stable and unstable manifolds of $M_{\varepsilon}$, smooth continuations of the corresponding manifolds of $M_{0}$, on which the flow is fast.

\section{Periodic solutions}

The existence and smoothness of the slow manifold, in combination with the possibility of a regular expansion describing the slow manifold drift, enables us to take a fairly easy shortcut to obtain periodic solutions. Note, that if we restrict ourselves to periodic solutions within a slow manifold, this excludes the case of nonhyperbolic transition as found in relaxation oscillations.

\subsection{Averaging in the slow manifold}

We will develop the following setup of a theorem leading to periodic solutions. Consider the autonomous system in $\mathbb{R}^{n} \times \mathbb{R}^{m} \times \mathbb{R}^{+}$

$$
\begin{aligned}
\dot{x} & =f_{0}(x, y)+\varepsilon f_{1}(x, y)+\varepsilon^{2} \cdots, \quad x \in D \subset \mathbb{R}^{n}, t \geq 0, \\
\varepsilon \dot{y} & =g_{0}(x, y)+\varepsilon g_{1}(x, y)+\varepsilon^{2} \cdots, \quad y \in G \subset \mathbb{R}^{m},
\end{aligned}
$$

where $f_{0}, f_{1}, g_{0}, g_{1}$ are $C^{1}$ vector functions, the dots represent bounded and smooth higher order terms. Furthermore the assumptions of Tikhonov's and Fenichel's theorems apply for $0 \leq t \leq L$.

For the solutions in the slow manifold we can apply the expansion (2) $y=\phi(x)+\varepsilon y_{1}(x)+$ $\varepsilon^{2} \cdots$ with $g_{0}(x, \phi(x))=0$. 
For $x(t)$ in the slow manifold this results in

$$
\dot{x}=f_{0}(x, \phi(x))+\varepsilon \frac{\partial f_{0}}{\partial y}(x, \phi(x)) y_{1}(x)+\varepsilon f_{1}(x, \phi(x))+\varepsilon^{2} \cdots .
$$

This is still a very general system and much depends on the explicit solvability of the reduced equation which arises for $\varepsilon=0$ or, more in general, on what is known about the reduced equation. Note, that if we would strictly apply the O'Malley-Vasil'eva expansion for the equations governing the slow manifold flow, this may produce secular terms when approximating periodic solutions. Using eq. (3), secular terms can be avoided.

We will give an example of a more specific result leading to periodic solutions. Using the wealth of results on periodic solutions by averaging and normalisation, it is not difficult to develop this idea to other cases.

Assume that the autonomous system above is of the form

$$
\begin{aligned}
\dot{x} & =A(y) x+\varepsilon f_{1}(x, y)+\varepsilon^{2} \cdots, \quad x \in D \subset \mathbb{R}^{n}, t \geq 0, \\
\varepsilon \dot{y} & =g_{0}(x, y)+\varepsilon g_{1}(x, y)+\varepsilon^{2} \cdots, \quad y \in G \subset \mathbb{R}^{m},
\end{aligned}
$$

with $f_{1}, g_{0}, g_{1}$ smooth vector functions, $A(y)$ a $n \times n$-matrix with smooth entries, the dots represent bounded and smooth higher order terms. For simplicity we assume that we have shifted the root of $g_{0}(x, y)=0$ such that $g_{0}(x, 0)=0$; this is not a necessary restriction as is shown by example 8.10 in [22]. Furthermore the assumptions of Tikhonov's and Fenichel's theorems apply with

$$
\text { Re Sp } \frac{\partial g_{0}}{\partial y}(x, 0) \leq-\mu<0, x \in D, 0 \leq t \leq L .
$$

If the matrix $A(0)$ has a spectrum with purely imaginary eigenvalues and can be diagonalised, we can perform periodic or quasi-periodic averaging or apply the Poincaré-Lindstedt method. Putting $\varepsilon=0$, the solutions of the reduced equation $\dot{x}=A(0) x$ are determined by a fundamental matrix which is quasi-periodic or T-periodic.

For the slow manifold approximation, the expansion (2) becomes $y=\varepsilon y_{1}(x)+\varepsilon^{2} \cdots$, leading to

$$
y_{1}(x)=-{\frac{\partial g_{0}}{\partial y}}^{-1}(x, 0) g_{1}(x, 0) .
$$

The behaviour of $x(t)$ in the slow manifold is governed by the equation

$$
\dot{x}=A(0) x+\varepsilon A\left(y_{1}(x)\right) x+\varepsilon f_{1}(x, 0)+\varepsilon^{2} \cdots .
$$

In the T-periodic case (condition $T<L$ ), we can apply to eq. (4) the Poincaré-Lindstedt method, in general we can apply averaging. If the averaged vector field in the T-periodic case has a critical point with only one eigenvalue with real part zero, we can apply the Bogoliubov-Mitropolsky theorem to obtain the existence and approximation of a periodic solution; for examples and proofs of the Poincaré-Lindstedt and the Bogoliubov-Mitropolsky theorems see [20]. 


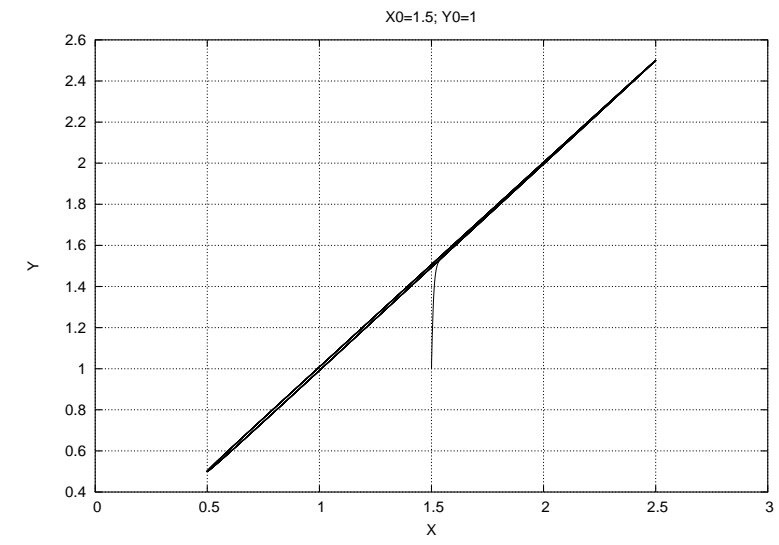

Figure 1: Solution approaching the periodic solution in the slow manifold of the modified logistic equation (5) with $x(t)=1.5+\sin t, x(0)=1.5, y(0)=1, \varepsilon=0.01$.

\section{$2.2 \quad$ Examples}

The ideas of the preceding subsection are illustrated by the following example.

\section{Example 2.1}

Consider the system

$$
\begin{aligned}
\ddot{x}+x & =\mu\left(1-x^{2}\right) \dot{x}+\nu f(x, \dot{x}, y)+\varepsilon^{2} \cdots, \\
\varepsilon \dot{y} & =-g(x, \dot{x}) y+\varepsilon h(x, \dot{x}, y)+\varepsilon^{2} \cdots,
\end{aligned}
$$

in which $\mu, \nu$ are constants, $\mu>0, f, g, h$ are smooth scalar functions; $g(x, \dot{x})$ is a positive function, bounded away from zero.

The behaviour of $x(t)$ in the slow manifold is governed by

$$
\ddot{x}+x=\mu\left(1-x^{2}\right) \dot{x}+\nu f\left(x, \dot{x}, \varepsilon \frac{g(x, \dot{x})}{h(x, \dot{x}, 0)}\right)+\varepsilon^{2} \cdots .
$$

Assume first that the constant $\mu$ is independent of $\varepsilon, \nu=\varepsilon$, then the equation obtained by putting $\varepsilon=0$ is the van der Pol-equation without small parameter. This equation contains a structurally stable periodic solution and for instance Anosov's (1963) theorem applies to obtain a nearby periodic solution of the original system.

Assume now that $\mu=\nu=\varepsilon$ or $\mu=\varepsilon, \nu=\varepsilon^{2}$. In these cases Anosov's (1963) theorem (and any other classical theorem) does not apply. If $\mu=\nu=\varepsilon$, the approximating equation is

$$
\ddot{x}+x=\varepsilon\left(1-x^{2}\right) \dot{x}+\varepsilon f(x, \dot{x}, 0)+\varepsilon^{2} \cdots .
$$

We can average the equation and if we find a critical point with one eigenvalue with real part nonzero, we can apply the Bogoliubov-Mitropolsky theorem to obtain the existence and approximation of a periodic solution. 
If $\mu=\varepsilon, \nu=\varepsilon^{2}$, the approximating equation is to $O\left(\varepsilon^{2}\right)$ the van der Pol-equation with a small parameter. This immediately leads to the existence of a nearby periodic solution in the slow manifold.

If we have apriori knowledge of the behaviour of the slow solution $x(t)$, it is easier to obtain results.

Example 2.2

Consider the scalar equation

$$
\varepsilon \dot{y}=x(t) y-y^{2},
$$

in which $x(t)$ is determined by a differential equation or is explicitly given. The equation for $y$ is the modified logistic equation, modified in the sense that the growth rate is varying. One can put $t / \varepsilon=\tau$, then denote time again by $t$ to obtain the equation

$$
\dot{y}=x(\varepsilon t) y-y^{2} \text {. }
$$

This equation represents for $y \geq 0$ population changes with growth rate $x$, slowly varying in time.

For $y \geq 0$ the first order appoximation of the slow manifolds are $y=0$ (also exact solution) and $y=x$. To apply Fenichel theory, assume that $0<\alpha \leq x(t) \leq \beta$ with positive constants $\alpha$ and $\beta$. In this case $y=0$ is unstable, the slow manifold corresponding with $y=x$ is stable. Using the expansion $y=x+\varepsilon y_{1}(x)+\varepsilon^{2} \cdots$, the approximation for the relation between $y$ and $x$ in the stable slow manifold becomes

$$
y(t)=x(t)-\varepsilon \frac{\dot{x}(t)}{x(t)}+\varepsilon^{2} \cdots .
$$

As an example we choose

$$
x(t)=1.5+\sin t
$$

leading to the approximation of the periodic solution

$$
y(t)=1.5+\sin t-\varepsilon \frac{\cos t}{1.5+\sin t}+\varepsilon^{2} \cdots .
$$

The solutions will approach the periodic solution exponentially fast. The behaviour of such a solution is pictured in fig. 1 .

\section{Nonhyperbolic transitions}

Transitions arising from nonhyperbolicity have been studied in various contexts. For an interesting boundary value problem and references, see [13].

\subsection{Relaxation oscillations}

A classical phenomenon are relaxation oscillations where jumps, fast transitions, take place after moving along a slow manifold that becomes unstable. For this topic see [10], [14], [17] and chapter 4 in [2]. Most rigorous analysis is carried out for two-dimensional autonomous and forced problems and it is not easy to extend this to more dimensions. We discuss briefly a four-dimensional problem from [21] where the evidence is partly numerical. 


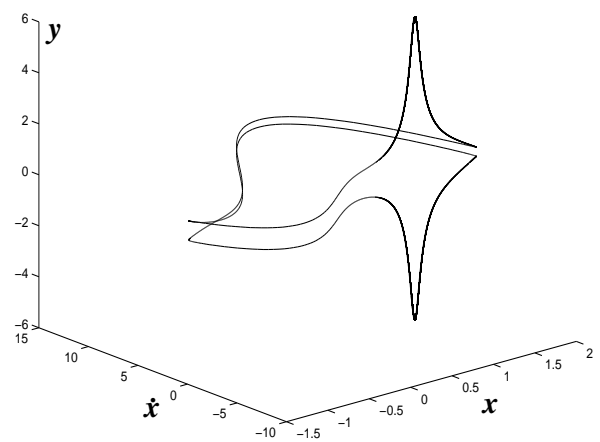

Figure 2: A periodic limit set of system (6) for $\mu=10, c=-2.2, d=0.03, \kappa=0.075$ with high starting values of the $y$-oscillation. Transient orbits are left out. The stable part of the slow manifold is present near the extreme values of $y$.

\section{Example 3.1}

Consider the system of coupled oscillators

$$
\begin{aligned}
\ddot{x}+x & =\mu\left(1-x^{2}\right) \dot{x}+\mu c \dot{x} y^{2}, \mu \gg 0, \\
\ddot{y}+\kappa \dot{y}+q^{2} y & =d x y .
\end{aligned}
$$

The quantity $1 / \mu$ plays the part of $\varepsilon$. In generalised Liénard variables, system (6) becomes

$$
\begin{aligned}
\frac{1}{\mu} \dot{x} & =z+x-\frac{1}{3} x^{3}+c x y^{2}, \\
\dot{z} & =-\frac{1}{\mu} x-2 c x y \dot{y}
\end{aligned}
$$

with the equation for $y$ added. The slow manifold in 4-space is given by

$$
z=-\left(1+c y^{2}\right) x+\frac{1}{3} x^{3},
$$

which is unstable if $1+c y^{2}-x^{2}>0$. The slow manifold corresponds with a 3 -dimensional cubic cylinder parallel to the $\dot{y}$-axis.

Consider the dynamics of the system with parameter values $c=-2.2, d=0.03, \kappa=0.075$, for solutions starting at some distance from the $y, \dot{y}$-coordinate plane. Leaving out the transient, we find a periodic limit set illustrated in fig. 2 ; this is a projection in 3 -dimensional space. Projecting the limit set on the $x, \dot{x}$-plane we find a strongly perturbed relaxation oscillation, see fig. 3. For comparison the unperturbed relaxation oscillation (coupling $c=0)$ is indicated by dots.

In the system we have coexisting attractors. We find also a chaotic attractor with KaplanYorke dimension $2.3 \cdots$. For details see [21]. 


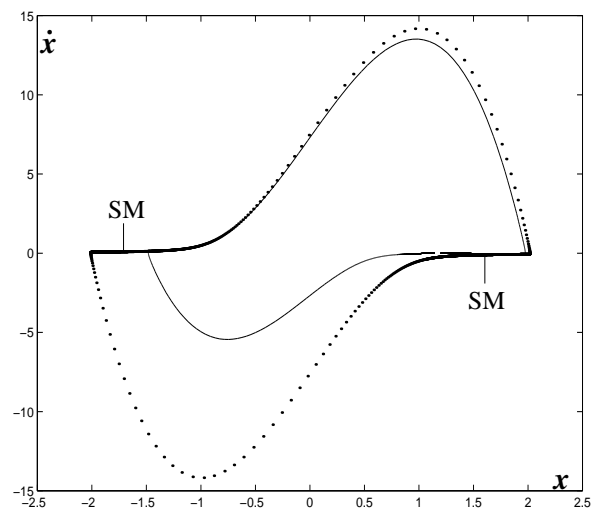

Figure 3: A periodic limit set of system (6) for $\mu=10, c=-2.2, d=0.03, \kappa=0.075$ with high starting values of the $y$-oscillation, projected on the $x-\dot{x}$ plane. The dotted orbit corresponds with the unperturbed relaxation oscillation. In the perturbed state the motion on the slow manifolds is reduced and the limit cycle becomes asymmetric. $S M$ is the stable part of the slow manifold.

\subsection{Canards}

Canard solutions play a special part. We shall use the following description.

Canard solutions are bounded solutions that, starting near an attracting normally hyperbolic slow manifold, cross a singularity of the system of differential equations and follow for an $O(1)$ time a normally hyperbolic repelling slow manifold.

Note that, depending on the dimension of the problem and the nature of the singularity, the description usually has to be more specific.

The first example of such behaviour was found by the Strassbourg group working in nonstandard analysis for a perturbed van der Pol-equation; see for details and references [4]. In this first case, the singularity crossed is a fold point. In [16], second order slow-fast systems have been analysed for homoclinic bifurcations; it contains a population dynamics application with canard-like behaviour. This sticking to a repelling manifold is discussed in a general context in [19] where it is called 'delay in loss of stability'. In [15] transitions through transcritical and pitchfork singularities are analysed.

We will discuss an example of transition through a transcritical singularity, the logistic canard, that can be calculated explicitly.

\section{Example 3.2}

Consider the modified logistic equation (5) for $y \geq 0$

$$
\varepsilon \dot{y}=x(t) y-y^{2},
$$

in which the growth rate $x(t)$ can now take positive and negative values. We can solve the 


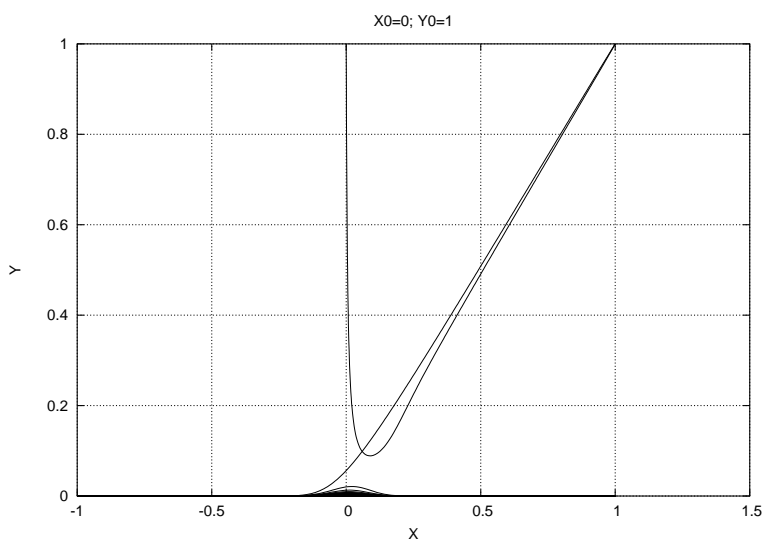

Figure 4: Solution approaching the periodic solution in $y=0$ of the modified logistic equation (5) with $x(t)=\sin t, x(0)=0, y(0)=1, \varepsilon=0.01$. To the right of the $y$-axis, $y=0$ corresponds with an unstable slow manifold.

equation for general continuous $x(t)$ and $y(0)=y_{0}>0$ :

$$
y(t)=\frac{e^{\frac{1}{\varepsilon} \int_{0}^{t} x(s) d s}}{\frac{1}{y_{0}}+\frac{1}{\varepsilon} \int_{0}^{t} e^{\frac{1}{\varepsilon} \int_{0}^{s} x(u) d u} d s} .
$$

If $x(t)$ is $T$-periodic we can write

$$
x(t)=a+f(t)
$$

with $a$ a real constant and $f(t)$ a zero average continuous function. In the cases that $x(t)$ is quasi- or almost-periodic we can write similar expressions.

Assuming that $x(t)$ changes sign and is $T$-periodic, we have two cases.

Case 1: $a \leq 0$. We state that $\lim _{t \rightarrow \infty} y(t)=0$. In the extended $(x, y)$ system, the only periodic solution is the trivial solution $x=x(t), y=0$. If $a<0$ the proof is simple: multiplying with $\exp (-a t / \varepsilon)$ produces a bounded numerator and a monotonically increasing denominator. A similar reasoning applies if $a=0$. In this case $y(t)$ also decreases exponentially; this can be seen by estimating a lower bound of the integral in the denominator by restricting to the time-intervals where $f(t)$ is positive. In fig. 4 we took $x(t)=\sin t$ for an illustration.

Case 2: $a>0$ with $a+\min _{0 \leq t \leq T} f(t)<0$. In this case a canard-like periodic solution exists. Several proofs are possible, but a simple one runs as follows.

Assuming that $y(0)=y(T)=y_{0}(>0)$, we obtain from the solution (8) the expression

$$
y_{0}=\frac{e^{\frac{a T}{\varepsilon}}-1}{\frac{1}{\varepsilon} \int_{0}^{T} e^{\frac{1}{\varepsilon} \int_{0}^{s}(a+f(u)) d u} d s} .
$$

The righthand side being positive, we have constructed a positive solution for $y_{0}$ and so a positive periodic solution.

For more explicit choices of $x(t)$ we can show that $y_{0}$ can be exponentially small and can be $O(1)$. Consider for instance the case $x(t)=0.5+\sin t$, see fig. 5 . 


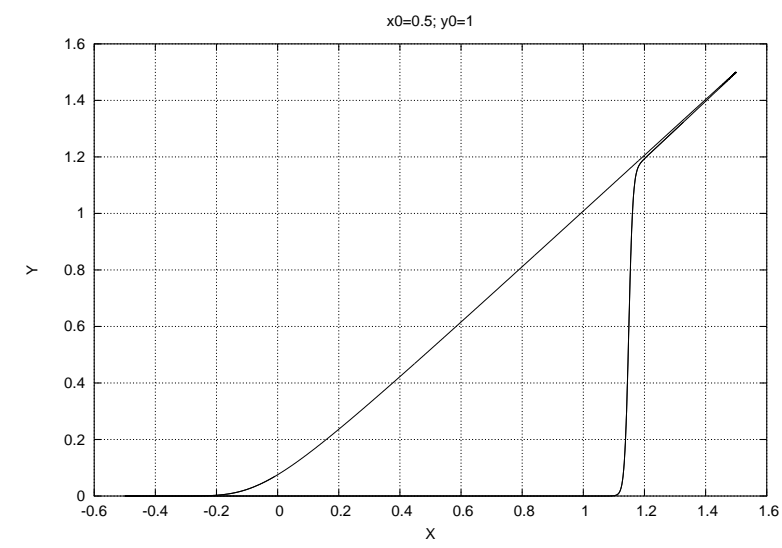

Figure 5: Canard-like periodic solution of the modified logistic equation (5) with $x(t)=$ $0.5+\sin t, \varepsilon=0.01$. To the right of the $y$-axis, $y=0$ corresponds with an unstable slow manifold.

It is of interest to know whether this canard-like behaviour of the solutions of the modified logistic equation (5) persist if the growth rate function $x(t)$ is quasi- or almost-periodic. Most of the results of the periodic case carry over, although the arguments are more complicated. A highly nontrivial case arises if we have an almost-periodic function with a spectrum that is not bounded away from zero. If the generalised average of such a function vanishes, it may still have an unbounded primitive. Consider the almost-periodic function with zero mean average

$$
f(t)=\sum_{n=0}^{\infty} \frac{1}{(2 n+1)^{2}} \sin \left(\frac{t}{2 n+1}\right) .
$$

In [22], appendix 15.8, the growth rate with time of the integral of this function is estimated. Remarkably enough it has been shown in [3], that the canard-like behaviour of the solutions of equation (5) persists for such functions. For an example using the function (9), see fig. 6 ; the figure shows sudden population growth at irregular times, triggered off by the almost-periodic function.

\section{Discussion}

In section 2 we discussed the existence of periodic solutions within slow manifolds. The idea used here, is related to the much older analysis of the dynamics in center manifolds. It is rather straightforward to extend these results to existence results for tori within slow manifolds of dimension 3 or higher.

The modified logistic equation (5) with alternating negative and positive growth rates is a simple metaphor for more complex models. It is interesting that the solutions of this equation show sudden 'population explosions' related to canard behaviour.

Both slow manifolds as discussed in this paper and resonance manifolds in dynamical sys- 


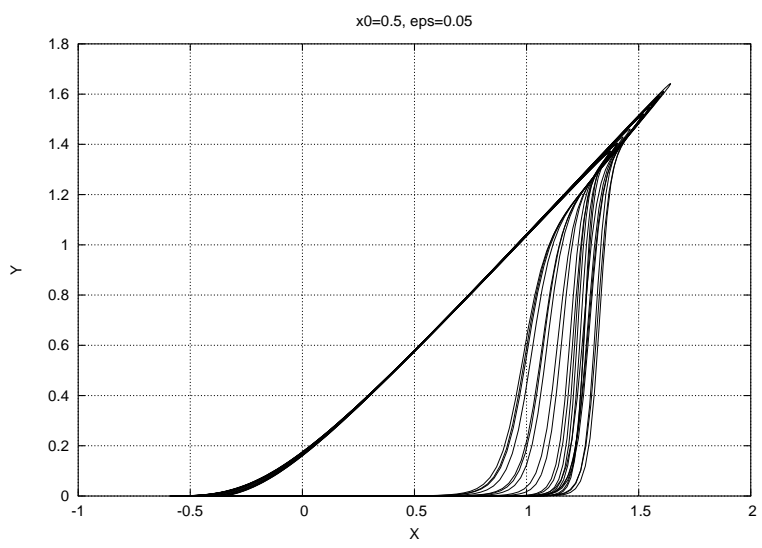

Figure 6: Canard-like periodic solution of the modified logistic equation (5) with $x(t)=$ $0.5+f(t), \varepsilon=0.05$ with $f(t)$ the almost-periodic function $(9) ; 0 \leq t \leq 150$.

tems represent slow-fast dynamics. However the similarity is superficial as in the equations for resonance manifolds nonhyperbolic features are so typical that a different approach is needed.

\section{Acknowledgement}

Section 2 grew out of a discussion with Arjen Doelman; also remarks and references by Peter Szmolyan and Yuri Kuznetsov on canards were useful. There were many stimulating discussions with Taoufik Bakri who made most of the pictures of this paper.

\section{References}

[1] D.V. Anosov, On limit cycles in systems of differential equations with a small parameter in the highest derivatives, AMS Translations, Series 2, 33 (1963) pp. 233-276.

[2] V.I. Arnol'd, V.S. Afrajmovich, Yu.S. Il'yashenko and L.P. Shil'nikov, Bifurcation Theory, in Dynamical Systems V (V.I. Arnol'd, ed.), (1994) Springer-Verlag.

[3] T. Bakri, On a modified logistic equation, JDEA, to be published.

[4] W. Eckhaus, Relaxation oscillations including a standard chase on French ducks, in Asymptotic Analysis II, Lecture Notes in Mathematics 985 (1983) pp. 449-494.

[5] N. Fenichel, Persistence and smoothness of invariant manifolds for flows, Ind. Univ. Math. J., 21, (1971) pp. 193-225.

[6] N. Fenichel, Asymptotic stability with rate conditions, Ind. Univ. Math. J., 23 (1974) pp. 1109-1137. 
[7] N. Fenichel, Asymptotic stability with rate conditions, II, Ind. Univ. Math. J., 26 (1977) pp. 81-93.

[8] N. Fenichel, Geometric singular perturbations theory for ordinary differential equations, J. Diff. Eq., 31 (1979) pp. 53-98.

[9] L. Flatto and N. Levinson, Periodic solutions of singularly perturbed systems, J. Rat. Mech. Analysis, 4 (1955) pp. 943-950.

[10] J. Grasman, Asymptotic Methods for Relaxation Oscillations and Applications, Springer-Verlag, New York (1987) 222 pp.

[11] C.K.R.T. Jones, Geometric Singular Perturbation Theory, in R. Johnson, ed., Dynamical Systems, Montecatini Terme, Lecture Notes in Mathematics 1609, Springer-Verlag, Berlin (1994) pp. 44-118.

[12] T.J. Kaper, An introduction to geometric methods and dynamical systems theory for singular perturbation problems, in Jane Cronin and Robert E. O'Malley, Jr., eds., Analyzing multiscale phenomena using singular perturbation methods, Proc. Symposia Appl. Math, AMS 56 (1999) pp. 85-131.

[13] CN. Kopell and S.V. Parter, A complete analysis of a model nonlinear singular perturbation problem having a continuous locus of singular points, Adv. Appl. Math. 2, (1983) pp. 212-238.

[14] M. Krupa and P. Szmolyan, Relaxation oscillation and canard explosion, J. Diff. Equations 174, (2001) pp. 312-368.

[15] M. Krupa and P. Szmolyan, Extending slow manifolds near transcritical and pitchfork singularities, Nonlinearity 14, (2001) pp. 1473-1491.

[16] Yu.A. Kuznetsov, S. Muratori and S. Rinaldi, Homoclinic bifurcations in slow-fast second order systems, Nonlinear Analysis, Theory, Methods \& Applications 25, (1995) pp. $747-762$.

[17] P. Szmolyan and M. Wechselberger, Relaxation oscillations in $\mathbb{R}^{3}$, J. Diff. Equations 200, (2004) pp. 69-104.

[18] A.N. Tikhonov, Systems of differential equations containing a small parameter multiplying the derivative, Mat. Sb. 31 (1952) pp. 575-586.

[19] A.I. Neihstadt, Asymptotic investigation of the loss of stability by an equilibrium as a pair of imaginary eigenvalues slowly cross the imaginary axis, Usp. Mat. Nauk 40 (1985) pp. 190-191.

[20] F. Verhulst, Nonlinear Differential Equations and Dynamical Systems, Springer-Verlag, New York (2000) 304 pp.

[21] F. Verhulst and Abadi, Autoparametric resonance of relaxation oscilations, ZAMM 85 (2005) pp. 122-131.

[22] F. Verhulst, Methods and applications of singular perturbations, boundary layers and multiple timescale dynamics, Springer-Verlag (2005) 340 pp. 